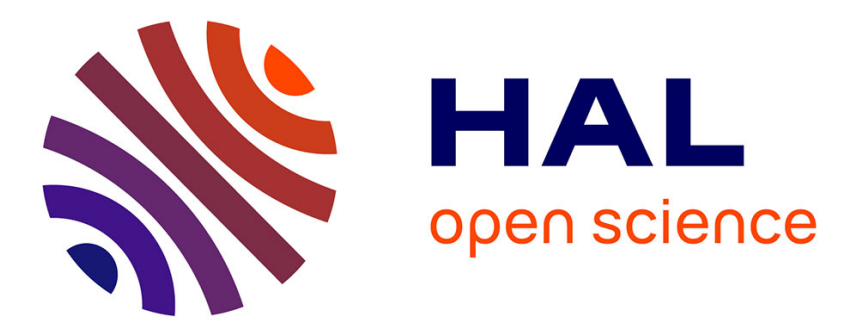

\title{
Crise de plume et souveraineté du pinceau. Écrire la peinture de Diderot à Proust by Nicolas Valazza \\ Loïse Lelevé
}

\section{To cite this version:}

Loïse Lelevé. Crise de plume et souveraineté du pinceau. Écrire la peinture de Diderot à Proust by Nicolas Valazza. MLN, 2014, pp.1070 - 1077. 10.1353/mln.2014.0089 . hal-01867870

\section{HAL Id: hal-01867870 \\ https://hal.science/hal-01867870}

Submitted on 14 Sep 2018

HAL is a multi-disciplinary open access archive for the deposit and dissemination of scientific research documents, whether they are published or not. The documents may come from teaching and research institutions in France or abroad, or from public or private research centers.
L'archive ouverte pluridisciplinaire HAL, est destinée au dépôt et à la diffusion de documents scientifiques de niveau recherche, publiés ou non, émanant des établissements d'enseignement et de recherche français ou étrangers, des laboratoires publics ou privés. 
«Crise de plume et souveraineté du pinceau. Écrire la peinture de Diderot à Proust by Nicolas Valazza », MLN, Volume 129, Number 4, September 2014 (French Issue), pp. 1070-1077 (Review)

Loïse Lelevé

Published by Johns Hopkins University Press

DOI: $10.1353 / m \ln .2014 .0089$

Nicolas Valazza. Crise de plume et souveraineté du pinceau. Écrire la peinture de Diderot à Proust. Paris : Classiques Garnier, coll. "Études romantiques et dix-neuviémistes », 2013. 357 pages. ISBN 978-2-8124-0863-2

C'est par une double exergue que s'ouvre l'ouvrage que Nicolas Valazza a tiré de sa thèse de doctorat soutenue à l'université Johns Hopkins ${ }^{1}$ : par une citation de George Bataille, d'abord, sur un "art que ne guide plus l'autorité », et par un passage de la Crise de vers de Stéphane Mallarmé, à qui, tout absent qu'il soit de son corpus d'étude, il rend hommage dans son titre et dont il reprend le diagnostic d'une « exquise crise» de la littérature, pour en faire une « crise de plume ». On perçoit dès lors toute l'originalité de son étude qui consiste à mettre en valeur l'interdépendance de ces deux mouvements qui marquèrent la littérature du long dix-neuvième siècle qui intéresse N. Valazza : l'émancipation de la peinture des divers discours d'autorité mis en place depuis la Renaissance et ce qu'il appelle " un régime critique de l'écriture », à travers lequel les écrivains se voient contraints d'interroger leur propre pratique devant l'affirmation de ce qu'ils en viennent à percevoir comme une peinture souveraine. Car ce n'est ni à l'histoire de l'autonomisation du champ pictural, ni à une analyse diachronique de la constitution d'un discours critique sur la peinture, que prétend l'auteur. Les termes de «souveraineté » et de "plume» sont loin d'être contingents : ils indiquent dès le seuil de l'ouvrage que ce dont il s'agit, profondément, c'est de la confrontation des écrivains à une peinture dont ils s'aperçoivent qu'elle est devenue irréductible au discours, qu'elle échappe à toute tentative de mise en mot. C'est ainsi la faillite d'une approche théorique classique de l'art, fondée sur l'ut pictura poesis, qui provoque la nécessité de l'instauration d'un régime critique de l'écriture, pour «combler la faille sémantique creusée par le coup de pinceau », sans nier «l'altérité fondamentale du geste du peintre » (13). À partir de Chardin, la peinture ne peut plus être appréhendée selon les catégories hiérarchiques traditionnelles : entre sujétion et fascination, les huit écrivains étudiés par l'auteur, tous également critiques d'art - Diderot, Balzac, Baudelaire, les Goncourt, Zola, Huysmans, Proust -, doivent réinterroger les modalités et les formes de leur propre écriture. Ce qui ne se fait pas sans tensions : à l'opposé de la tendance de nombreux critiques à voir dans la littérature du $\mathrm{XIX}^{\mathrm{e}}$ siècle la supplantation de la théorie des «arts sœurs » par l'alliance fraternelle entre

\footnotetext{
${ }^{1}$ On en trouvera un compte-rendu critique par Élise Pavy Guilbert, in «Nicolas Valazza, Crise de plume et souveraineté du pinceau, Écrire la peinture de Diderot à Proust, Pierre Glaudes et Paolo Tortonese (Éds.), Études romantiques et dix-neuvièmistes, 35, 2013, 357 p. ISBN 9782812408632 », Recherches sur Diderot et sur l'Encyclopédie 1/ 2013 ( $\mathrm{n}^{\circ}$ 48), p. 309-314, URL : www.cairn.info/revue-recherches-sur-diderot-et-sur-lencyclopedie-2013-1-page-309.htm.
} 
l'écrivain et le peintre, N. Valazza souhaite, lui, étudier les frictions entre les deux. Le critique s'efforce donc de traquer les symptômes de cette crise dans les œuvres des écrivains, mais aussi d'en retracer les causes et l'évolution dans le parcours littéraire de chacun d'eux, depuis son instauration à travers le rapport fasciné de Diderot aux natures mortes de Chardin à sa dissolution devant la souveraineté de la métaphore proustienne. Entre les deux, si une progression se dessine bel et bien, et si ses contours sont, sous certains aspects, chronologiques, elle est pourtant loin d'être linéaire : de Diderot à Proust, chapitre par chapitre et auteur par auteur, tant la souveraineté du pinceau que la crise de plume auront été mises en cause aussi bien que reconnues, constatées ou outrepassées.

Le premier à se trouver confronté, dans sa tentative de descriptions des œuvres du Salon, à un besoin de renouvellement des concepts devant l'image, est Denis Diderot, réduit à la « stupéfaction », «l'admiration silencieuse » (34) devant l'œuvre de Chardin : le critique, qui n'a plus d'idées à débattre, ne peut que se taire, devant un artiste chez qui l'assomption de la technique s'effectue au détriment du sujet dont elle rachète la trivialité. Chardin renverse ainsi la hiérarchie classique des arts et dévalue les discours esthétiques qu'elle régit. Diderot en réponse ne peut que développer la notion de style, qui déplace l'intérêt de la critique du genre et de l'adéquation aux règles à la prise en compte de la singularité de l'œuvre : se dessinent ainsi à la fois une nouvelle visibilité de l'œuvre et une nouvelle approche critique, bien que Diderot continue de reconnaître "l'empire du texte sur l'image» (45). Il tend encore à rejeter une peinture qui, si elle n'est pas l'expression d'une idée ou d'une maxime, risque d'être muette : devant la peinture de Chardin en effet, Diderot en semble réduit à l'énumération nominale, incapable de former une phrase complète, donc d'accéder au niveau de la production du sens. Il ne lui reste alors que l'insistance sur l'indiscernabilité de la peinture et de la nature. Toutefois, en dehors des tableaux de Chardin, devant les toiles de Greuze par exemple, la peinture est chez lui une source inépuisable de création verbale, qui permet à Diderot de creuser « un espace de fiction au-delà de la surface peinte » (48), faisant ainsi vaciller, dès le début, les contours du genre de la critique d'art naissante. Une telle liberté d'interprétation, qui allie critique et fiction dans un même geste scriptural, ne sera pleinement retrouvée qu'avec Proust : c'est donc au moment de la constitution et de la dissolution du paradigme critique de l'écriture d'art que se joue et se déjoue pleinement la question des genres. En ce qui concerne Diderot, cependant, la menace d'une souveraineté de la peinture sur la littérature serait ce qui le pousse à ne pas prolonger le geste de reconnaissance critique de la vérité dans la peinture de Chardin qu'il esquisse dans le Salon de 1765. C'est pourtant cette même peinture, cette «cuisine» (64) de la chair et de la couleur, qui consacrera, le XIX ${ }^{\mathrm{e}}$ siècle venu, la souveraineté de la peinture.

Car la peinture ne cessera plus, alors, de remettre en cause le principe de représentation mimétique au fondement de l'ut pictura poesis et, partant, de la possibilité d'appréhender la peinture dans le discours. La menace d'une dissolution de la figure sous le poids du travail de la couleur est ainsi thématisée chez Balzac déjà, à travers le personnage de Frenhofer, héros du Chef-d'œuvre inconnu. Celui-ci fantasme en effet l'abolition de la « dissociation ontologique» 
(69) entre l'être et sa représentation à l'œuvre dans le régime mimétique pour « inclure la nature dans l'art » (70); la représentation de la chair se donnant ainsi pour but de dépasser le stade du visible et de la figure pour montrer «l'intimité de la forme», dans les termes de Merleau-Ponty que convoque abondamment le critique (71); quitte à défigurer l'image peinte dans le travail de cette "chair à vif, saisie de convulsions, celles du peintre se répercutant sur le tableau qu'il exécute » (73). L'affirmation de la souveraineté de l'art est ainsi indissociable de la hantise de sa fin, et inséparable de la création d'une "nouvelle école du regard " capable de soutenir la «vision haptique» (77) qu'exige le tableau, une vision capable, devant la distance abolie, d'abandonner les codes de la représentation mimétique au profit de la présentation de la chair et des structures du désir: la peinture, telle que l'envisagent Frenhofer et son héritier proclamé, Cézanne, se fait travail de la sensation, lie désormais indissolublement forme sensible et expression de la chair. Elle se rend ainsi irréductible à toute entreprise de verbalisation. Mais cette prédominance de la peinture n'existe, dans la diégèse, que sur un plan thématique: en réalité, le texte de Balzac demeure souverain. D'abord parce que le tableau est fictif, et que le récit s'instaure ainsi comme un pur acte de création indépendant de tout référent extérieur, y compris pictural ; ensuite parce que ce même texte finit par recouvrir le tableau de son discours, récupérant le tableau dans un dispositif linguistique qui, dans le moment même où il thématise sa souveraineté, le laisse inconnu.

De même, Baudelaire, à la suite de Balzac, ne remettra jamais véritablement en cause la théorie de l'ut pictura poesis. Son rapport à l'art s'établit à travers deux grandes tendances : d'une part, l'éloge de peintres, tel Delacroix, chez qui il perçoit un pouvoir d'évocation poétique au point de conférer à leur œuvre des valeurs métaphoriques qui fondent le commentaire qu'il donne de leur tableau. La « virtualité poétique de la couleur» est ainsi « dotée d'une autonomie signifiante » (117). La peinture est pensée comme irréductible au discours, mais tout, chez Baudelaire, est d'ordre littéraire : la réception des œuvres comme les termes dans lesquels elles sont présentées aux lecteurs. On retrouve ainsi sous sa plume des catégories comme le beau ou l'idéal ; mais elles sont devenues incertaines, minées par une crise esthétique qui est en fait, profondément, le symptôme d'une crise de sens face à ce que Baudelaire appelle «la modernité ». Devant l'accélération de l'histoire engendrée par celle-ci, modernité et idéal classique ne peuvent plus être pensés en adéquation, sans qu'il soit pour autant possible de renoncer à l'un ou à l'autre, d'où le sentiment de nostalgie qui imprègne toute son œuvre. L'autre grande tendance de son travail critique, devant le constat de la mort du dernier des "Phares", Delacroix, et avec lui de «la foi romantique dans "l'union des arts" » (130), sera de s'intéresser justement aux peintres de cette vie moderne qui a mis au jour «la caducité de l'Idéal» et le « caractère "bizarre" de la beauté » (128). Or « le poète de la vie moderne n' [est] de fait pas un peintre, mais un poète animé par le "désir de peindre" "(113). Son but est d'affronter la modernité et de «trouver la coloration symbolique de l'objet qu'il s'attache à peindre, afin de révéler son idéalité, sans renoncer pour autant à sa consistance matérielle » (134). Se met ainsi en place, à travers l'utilisation de l'allégorie, une esthétique de la dyschronie qui met en valeur le décalage entre la poursuite de l'idéal perdu et la prise en compte de la réalité moderne : la beauté 
ne peut ainsi plus être célébrée que mélancoliquement, dans sa perte. Une telle esthétique est de fait très éloignée de l'art pratiqué par Manet par exemple, un art de l'instant «agressif, dans la mesure où il se trouve vidé de toute fonction suggestive (poétique, dramatique, etc.) » (138). C'est cette menace brutale de l'inintelligibilité de l'art que tente de conjurer Baudelaire, à travers son entreprise d'allégorisation de la modernité, qui n'est en définitive qu'une entreprise de révélation de la dérive des signes et de la perte de sens dans le monde moderne. Le poète finit ainsi par marquer un écart irrémédiable entre la production picturale de ses contemporains et sa propre entreprise poétique.

Il faut donc attendre les Goncourt pour que la souveraineté de la peinture soit non seulement reconnue dans le discours, mais encore qu'elle affecte les structures mêmes de l'écriture qui reconnaît son autonomie et son pouvoir de fascination. C'est en affrontant à nouveau la peinture de Chardin que les Goncourt, dans le sillage de Diderot, vont chercher à « rendre visible, et plus généralement perceptible, ce qui était jusque-là passé inaperçu » dans l'art du peintre (145). Leur insistance, dans la monographie qu'ils lui consacrent, sur la matérialité de sa peinture confère à son œuvre une valeur ontologique, en ce qu'elle permettrait d'accéder à l'essence de la chose peinte à travers la perception synesthésique que ses tableaux provoquent. "C'est bien cette unité de sens (sémiotique et somatique), toute muette qu'elle soit, qui confère à la peinture de Chardin une "intensité de réalité" ». (149) Cette intensité est rendue possible, notamment, par le travail du coloris. Seule la couleur, promue comme fondamentalement insignifiante, est en effet apte à réaliser cette radicale rupture avec l'ut pictura poesis qu'entendent engager les deux frères : sa «cuisine» est à la fois ce qui assure la souveraineté du peintre et la possibilité d'une «écriture artiste ", d'un style visuel où le jeu des connotations vise, comme la peinture de Chardin telle que l'analysent les deux écrivains, à « tout peindre dans son ton vrai, sans rien peindre dans son ton propre » (153). La peinture n'est donc plus prise en compte pour son pouvoir d'évocation poétique mais pour la capacité, qu'elle partage avec l'écriture, sans pour autant confondre aucun de ses procédés avec elle, d'offrir une recréation phénoménique de la nature. C'est bien cette attention au phénomène dans toutes ses nuances qui pose un véritable défi à la plume des écrivains et les pousse à inventer une écriture attentive à la sensation et à la matérialité de l'objet. C'est aussi cette attention qui, in fine, aboutira à une mise en crise de leur écriture, à en croire N. Valazza pour qui la plume des Goncourt s'est en fait trouvée irrémédiablement assujettie au pinceau de Chardin, incapable, dans leur production de fiction, de "dépasser le stade de la "sensation", afin de s'inscrire dans une temporalité romanesque » (176). C'est là le paradoxe de leur œuvre : alors même qu'ils sont les premiers à reconnaître pleinement l'irréductibilité de l'acte pictural devant toute entreprise de récupération linguistique, ils ne parviennent ni à parachever cette intuition dans le cadre de leur production romanesque, ni à s'inscrire pleinement dans la modernité de leur époque : ils n'ont ainsi jamais reconnu la valeur de la peinture d'un Courbet ou d'un Manet.

Après Baudelaire et les Goncourt, tous réticents devant la production de tels illustres contemporains, c'est donc Zola qu'il faut attendre pour que la modernité picturale du XIX ${ }^{\mathrm{e}}$ siècle 
trouve une juste place dans les œuvres critiques et fictives des écrivains - encore que les rapports de Zola avec les impressionnistes et leurs successeurs, à commencer par Cézanne, ne cesseront de se compliquer avec le temps. Car la progression de ce que Zola nomme la "tâche » en peinture menace, en définitive, la figure : elle en estompe les contours, tend à la noyer sous la couleur. Zola promeut certes l'indifférence du sujet au profit du travail de la matière ; mais il ne peut renoncer à l'intelligibilité de la forme dont est garante la figure, intelligibilité qui semble encore pour lui être la condition de possibilité d'un discours sur la peinture. Il le peut d'autant moins que l'écriture naturaliste tend à renouer avec le fantasme d'une écriture transparente, capable d'exprimer la vérité des phénomènes qu'elle observe. Certes, dans L'CEuvre, une telle entreprise apparait indissociable d'un travail de la chair, emprunté au peintre, et que l'écrivain s'approprie, « transformant son laboratoire en une "sale cuisine" où il est forcé d'accoucher à grand-peine des œuvres de dégoût » (197). Mais l'écrivain peut encore sortir victorieux de cet éprouvant travail de la création ; le peintre, lui, est condamné à revivre, fatalement, le drame de Frenhofer. Celui-ci avait tenté de surmonter la dissociation ontologique à l'œuvre dans le processus de représentation en incluant la nature dans l'art, en s'appropriant la chair en peinture, quitte à défigurer son œuvre. Claude Lantier, lui, ne franchit pas ce pas du renoncement au figural; mais ce faisant, il se condamne à toujours vivre tragiquement la dépossession inhérente à toute représentation, l'incapacité de faire corps avec la peinture et de posséder la chair représentée. La peinture est donc terrifiante: soit elle comprend le risque de la perte du sens dans la progression de la «tâche ", soit elle se voue à un symbolisme qui s'écarte résolument du projet naturaliste et consomme la rupture entre la plume de l'écrivain, vivant et fidèle à son esthétique, et le pinceau du peintre. Cependant, tout n'est pas si tranché que ne voudrait le laisser entendre la fin du roman : $\mathrm{N}$. Valazza suggère ainsi que la description que donne le narrateur de la dernière toile de Claude est elle-même soumise à un processus analogique qui finit par faire dévier l'écriture, malgré les prétentions au naturalisme de l'auteur, vers un régime métaphorique; ceci sous l'influence de la peinture symboliste de Lantier et de ses équivalents historiques (Gustave Moreau par exemple), dont l'imaginaire érotique, fantasmatique, symbolique, viendrait hanter la plume de l'écrivain et compromettre son idéal d'objectivité et de transparence. En se trahissant ainsi elle-même, en trahissant en quelque sorte son propre projet, l'écriture en viendrait, in fine et malgré elle, à reconnaître la souveraineté de la peinture.

Il semble donc que la « crise de plume » se trouve confrontée, à la fin du XIX ${ }^{\mathrm{e}}$ siècle, à une impasse, qu'on trouvera thématisée sous la plume de Huysmans. D'une part, sous l'effet des mouvements décadents, la notion de modernité et de «modernisme », telle que l'aurait nommée le peintre, devient inadéquate pour appréhender la peinture contemporaine. D'autre part, l'entreprise scripturale de Huysmans le portera à dépasser pour la première fois un régime critique de l'écriture inapte à conférer un accès à une transcendance esthétique qu'il appelle de ses vœux. L'entreprise de critique moderniste avait pourtant semblé devoir promettre « un réel bonheur d'écriture " (236) : pour la première fois, un peintre, Degas, associait le travail de la chair non seulement à un art de la couleur mais aussi à un art du dessin. Or si la couleur avait été désignée comme ce qui devait toujours échapper à la plume, la promotion de la ligne, elle, permet 
une résurgence du graphisme en peinture, et donc une union renouvelée du peintre et de l'écrivain. Le bonheur sera pourtant de courte durée : la chair représentée par Degas est avilie, crispée, tordue, dans une nouvelle physiologie picturale qui bannit le désir au profit de l'exhibition de la décadence du siècle. Cette simultanéité de la mise en valeur de la chair et de son rejet, du désir et de la corruption, se retrouve dans l'entreprise esthétique du personnage de Des Esseintes, le protagoniste d'À Rebours. La défaite de la chair consacre le règne de l'ambivalence du symbole, transcendant à tous les arts et qui en refonde l'union. Mais cette adéquation nouvelle ne saurait être une traductibilité : le symbole n'est pas l'analogie (celle du « ut» de l'ut pictura poesis); ni fondamentalement littéraire, ni essentiellement pictural, il se constitue comme un « creux sémantique » (253), une énigme, comme les monstres difformes des peintres décadents, Moreau ou Redon. Difformité, creux : le symbole ne conduit pas à la plénitude de la création, mais est voué à la caducité. La souveraineté qu'il confère aux arts visuels, qui semblent grâce à lui échapper à toute entreprise iconologique, est appelée à être dépassée, non dans une entreprise de défiguration mais de transfiguration, que perçoit Huysmans dans la peinture religieuse « primitive », telle que le retable d'Issenheim en fournit l'exemple. Il n'y a dès lors plus de régime critique possible: la Révélation picturale, qui rend visible l'Incarnation, transcende à la fois toute histoire de l'art et toute interrogation critique sur l'écriture : ce sont et le modernisme et la crise de plume qui se trouvent ici dépassés par la promotion d'un nouveau régime esthétique, emprunt de contemplation religieuse.

Ce paradigme critique de l'écriture, qui avait largement marqué la production littéraire du $\mathrm{XIX}^{\mathrm{e}}$ siècle, se trouvera en fin de compte non plus seulement dépassé mais disséminé, dissout, sous la plume de Proust, au profit d'une textualité nouvelle qui remettra profondément en jeu les rapports du texte à l'image. Or, c'est en revenant à Chardin que Proust y parviendra : si celui-ci parvient à mettre en lumière les objets, à les faire accéder à une réalité artistique, le sens de ses natures mortes demeure opaque à l'écrivain. Aussi Proust s'ingéniera-t-il dans son entreprise critique à redonner vie aux natures mortes, à travers une « appropriation de la "chose" peinte en vue de sa resémantisation à des fins poétiques » (291). C'est toute l'entreprise de la Recherche qui se trouve en germe dans une telle démarche: la vision du tableau permet de percevoir «l'essence des choses » (294), que le narrateur du récit proustien s'efforce ensuite de retrouver dans et par l'écriture, où, insérées dans une temporalité romanesque - ce à quoi précisément avaient échoué les Goncourt - elles se réfléchissent sans cesse dans la mémoire du narrateur, et réfléchissent cette mémoire, au point de devenir l'un des fondements du récit. Car tout l'art de Proust consiste à dépasser l'impression, la présence de la chose délivrée du nom, telle que la présente la peinture de Chardin, ou, fictivement, celle d'Elstir. L'art de la "méta-figuration » d'Elstir (305), qui, par un jeu de métaphores visuelles, rend la figure irréductible à toute nomination, au profit d'une perception optique du réel, trouve son pendant scriptural dans la métaphore proustienne, outil d'incorporation du réel et de l'analogie à même l'écriture. Ainsi l'écriture proustienne mêle-t-elle linéarité de la narration et transcendance de cette même linéarité par le travail de la mémoire et la réalité de l'art, par le jeu des relations analogiques entre ses parties, par l'insertion de la vision du peintre et des voix multiples de l'historien, du romancier, 
du critique d'art, du théoricien: en somme, elle aboutit à l'établissement du Texte, tel que le théorisera le $\mathrm{XX}^{\mathrm{e}}$ siècle, Texte qui abolit les catégories génériques traditionnelles et par là-même le paradigme critique dont N. Valazza étudie toute l'évolution. Au terme de celle-ci, l'œuvre du peintre est ramené à l'état d'un motif textuel : elle n'est plus ce qui se pose comme défi, impasse ou énigme à l'écrivain, mais ce qui lui permet de construire une nouvelle relation du texte à l'image, au risque d'une «combinaison - voire une confusion - généralisée des signes » (324), qui nourrira toute la production littéraire du siècle/

L'ouvrage de Nicolas Valazza propose donc une exploration fascinante, rigoureuse et cohérente de ce régime critique qui mit en crise la littérature du XIX ${ }^{\mathrm{e}}$ siècle. On appréciera en particulier la solidité d'axes de réflexion transversaux qui confèrent au corpus déterminé par le critique toute sa solidité : la réflexion sur le rôle pictural de la chair, sur celui de peintres comme Chardin ou Moreau, qui viennent hanter plusieurs générations d'auteurs, mais aussi sur la double identité d'écrivain et de critique, permet d'appréhender la complexité de ce paradigme critique non seulement de manière linéaire, mais également synchronique. Cet ouvrage trouve donc une unité profonde à la fois dans l'identité des auteurs, dans les thèmes centraux de leurs œuvres, et les peintres souverains auxquels ils se confrontent. Certaines questions demeurent pourtant ouvertes à la fin de la lecture: par exemple, et quoique N. Valazza prenne grand soin de distinguer l'écriture critique des auteurs de leur production romanesque, on aimerait que soit posée de manière plus essentielle la question générique dans laquelle s'inscrit un tel régime d'écriture : le fait que celui-ci transcende les catégories génériques traditionnelles signifie-t-il pour autant qu'il engendre un même geste scriptural dans la production critique et celle de fiction? On pourrait s'interroger également sur le statut des œuvres d'art envisagées dans l'ouvrage : peut-on mettre sur le même niveau analytique des œuvres fictionnelles et des œuvres réelles, faut-il envisager une différence théorique entre elles pour rendre compte de leur présence textuelle ? On voudrait aussi presque voir ajouté un deuxième tome à cette recherche, qui interrogerait plus avant le rapport du langage lui-même à l'image, dans leurs spécificités opposées, même si ce n'est pas le sujet immédiat de N. Valazza. Toujours est-il que le parcours que propose cet ouvrage dans la littérature et la peinture du XIX ${ }^{\mathrm{e}}$ jette une lumière singulière sur la fraternité professée des peintres et des écrivains de cette époque, et nous encourage vivement à reconsidérer aussi bien leurs rapports que leurs œuvres.

LOÏSE LELEVÉ 